\title{
SPACE CHARGE EFFECT ON BETATRON OSCILLATIONS
}

\author{
K. M. Fung, C. M. Chu, W. C. Hsi, S. Y. Lee, Indiana University, Bloomington, IN \\ L. Ahrens, G. Smith, N. Williams, Brookhaven National Laboratory, Upton, NY
}

\begin{abstract}
The transverse motion of the beam at the AGS was studied by analyzing the coherent beam signals, digitized at a $10 \mathrm{~ns}$ interval, at various beam intensities. It was found that the coherent vertical tune shift of the centroid is consistent with the space charge model due to the image charge and current induced on the vacuum chamber wall. An enhanced linear coupling at a high intensity was also observed.
\end{abstract}

\section{INTRODUCTION}

The space charge effect is important for low and medium energy synchrotrons. The space charge force in high brightness beams can cause beam halo, beam envelope instability, beam emittance blow-up, collective beam instabilities, and more importantly beam loss. The space charge effect manifests itself in betatron and synchrotron tune shift, tune spreads, collective beam instability, etc.

The aim of this paper is to study the effect on betatron motion due to the space charge force. An initial excitation was given to the beam and then the sum and difference signals were measured (digitized) at different beam intensities below the threshold for instability. The coherent betatron motion was used to derive the betatron tune at different intensities. The coherent vertical tune shift of the centroid can then be obtained. The experimental data can be described by a model in which the beam induces image charge and current on the vacuum chamber wall whose electromagnetic field acts back on the beam.

The paper is organized as follows. In Sec. 2, we will discuss the experimental method which includes the excitation and measurement of the beam response and the observation of a few beam properties as a function of the beam intensity. In Sec. 3, we will give the details of data analysis, including a method to reduce the impact of longitudinal motion on the analysis of transverse motion. Finally, the conclusion will be given in Sec. 4.

\section{EXPERIMENTAL METHOD}

The AGS is a medium energy synchrotron with a circumference $807.12 \mathrm{~m}$. The horizontal and vertical betatron tunes are about 8.8 and 8.9 respectively. It can be considered as a machine with 60 FODO cells and 12 superperiods. The injector for the AGS is the AGS booster whose circumference is $1 / 4$ of the AGS ring. The single bunch beam intensity from the booster ranges from 1 to $20\left(\times 10^{12}\right)$ protons. This experiment involves a single bunch injected from the AGS booster at $1.9 \mathrm{GeV}$ kinetic energy.

To study the injection process with the space charge effect, we observe the betatron motion at the injection time.
Because of the intentional or unintentional offset between the injection orbit and the AGS closed orbit, betatron motion is induced. In other words, the excitation of the beam response is given in the form of an initial coherent transverse displacement. The sum and difference signals from a beam position monitor (BPM) was digitized at $10 \mathrm{~ns}$ per channel. We recorded 50002 channels in each injection process. This corresponds to 175 turns with a revolution period $T_{0}=2848 \mathrm{~ns}$. The experiment was carried out for the beam intensities $N_{\mathrm{B}}=2.7,3.9,4,5.7,6.5,13\left(\times 10^{12}\right)$ particles per bunch.

Fig. 1 shows a typical example of the sum signal for a beam intensity of $1.3 \times 10^{13}$ particles. Since the signals were digitized at $10 \mathrm{~ns}$, the longitudinal profile has a resolution of $10 \mathrm{~ns}$ and the total beam intensity can be obtained by integrating the sum signal. Since 175 turns corresponds to about $1 / 4$ of the synchrotron period, the beam bunch is evidently more tightly bunched at the end of the data.

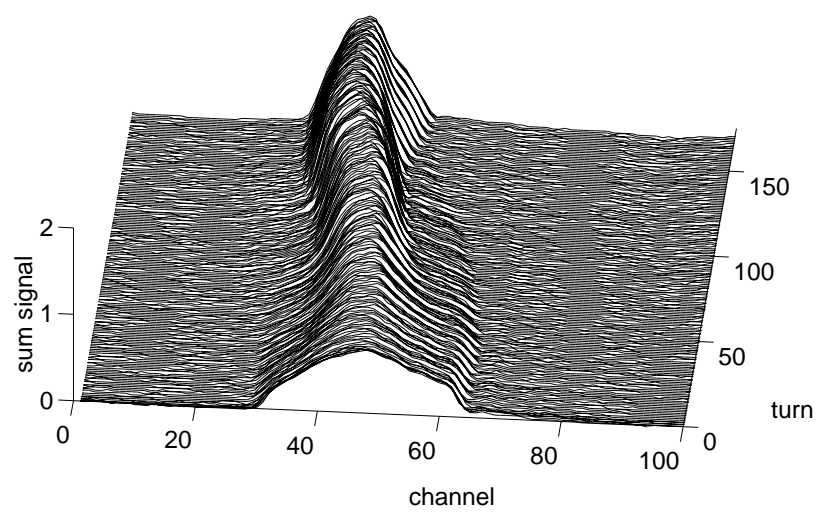

Figure 1: Evolution of the sum signal for $N_{\mathrm{B}}=1.3 \times 10^{13}$. Note that bunch length decreases.

Fig. 2 (middle,bottom) shows that the peak intensity of the sum signal and the integrated sum signal appear to be a linear function of the beam intensity derived from a current transformer. This means that the beam intensity has not yet saturated so that the peak intensity still depends on the beam current. In other words, all the beam intensities for the experiment are below the threshold for instability. Therefore our study of the space charge effect is justified.

\section{DATA ANALYSIS}

The data processing steps consist of background removal, signal normalization and tune shift computation. For the first step, the noise is assumed to follow a Gaussian distri- 


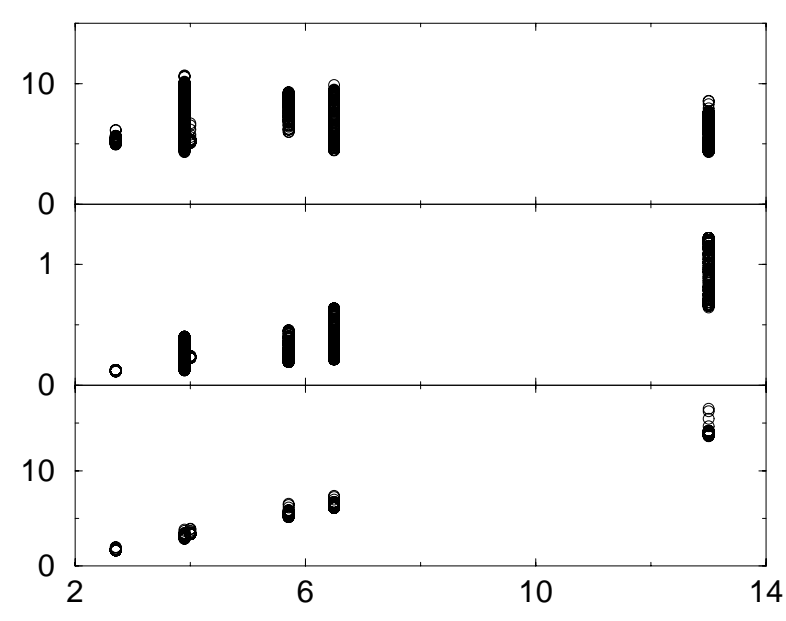

Figure 2: Beam properties at different intensities. The vertical width shows the fact that the beam properties vary with time. Horizontal axis shows intensity $N_{\mathrm{B}}\left(10^{12}\right)$ measured by a current transformer. The top plot shows the rms bunch length in ns, the middle plot shows the peak of the sum signal (in arbitrary unit), and the bottom plot shows the integrated sum signal (in arbitrary unit).

bution characterized by $\sigma$ and most of it could be removed by a cut at $\pm 3 \sigma$. Let $\Sigma$ and $\Delta$ be the sum and difference signals respectively. The normalized beam position signal is $y_{\mathrm{n}}=\Delta / \Sigma$. The actual beam position is given by $40 y_{\mathrm{n}}$ $\mathrm{mm}$, where the normalization factor $40 \mathrm{~mm}$ is the effective width of the BPM.

In order to study the transverse motion, we have to know the evolution of the transverse positions $x$ and $z$. If the bunch length were independent of time, then we can follow the transverse motion of each channel turn by turn without ambiguity. However, Fig. 2 (top) shows that the bunch length changes with time and therefore following the channels directly can be ambiguous. One way of following the channels turn by turn is to identify the channels which are $T_{0}$ apart and use linear interpolation to estimate the signal between the digitized data. This method has two difficulties. Firstly, not all particles in the bunch have the nominal revolution period $T_{0}$ due to the momentum spread $\Delta p / p$. The change in period $\Delta T_{0}$ is given by $[1,2]$

$$
\frac{\Delta T_{0}}{T_{0}}=\eta \frac{\Delta p}{p}
$$

where $\eta=1 / \gamma_{t}^{2}-1 / \gamma^{2}$ is the phase slip factor. Secondly, the synchrotron oscillation will affect $T_{0}$. A much better way to follow the transverse motion is to focus on the centroid. The centroid is the channel which always divides the beam longitudinally into two portions with the same number of particles. This method does not require constant bunch length and it does not rely on the nominal revolution period $T_{0}$ at all. An additional advantage of this method is that it completely eliminates the effect of the rigid longitu- dinal motion on the analysis of the transverse motion. This method can readily be generalized to include other channels. For example, we can select three channels in such a way that they always divide the beam longitudinally into four portions which contain the same number of particles. These channels represent the transverse motion of the head, the centroid and the tail of the beam. In general, we can label each channel by the fraction of the particles located in front of the channel. This labeling scheme combined with a linear interpolation between digitized data allows us to locate any channel longitudinally without ambiguity. For the above example, those three channels will be labeled as $25 \%, 50 \%$ and $75 \%$ respectively. The transverse motion for the above three channels is shown in Fig. 3.

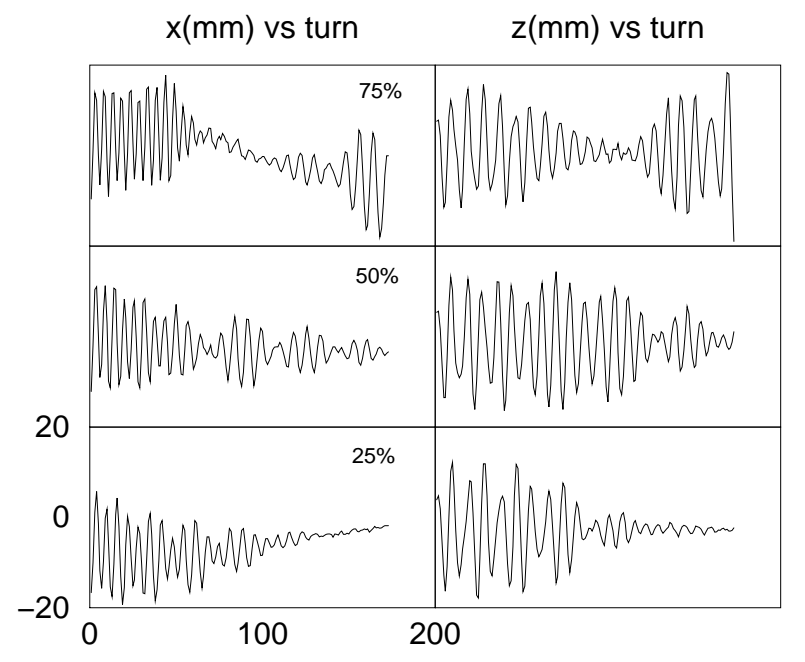

Figure 3: Betatron oscillations for $N_{\mathrm{B}}=1.3 \times 10^{13}$. Left: horizontal motion. Right: vertical motion. Bottom to top: the head, the centroid and the tail of the beam.

Fig. 3 (left: top,bottom) shows that the horizontal motions of the channels $25 \%$ and $75 \%$ have opposite trends after the first 50 turns. This is related to a decrease in the bunch length which can be seen in Fig. 1 due essentially to a mismatched synchrotron phase space during the injection. The tune shift due to the coupling of transverse motion can also be seen. For the last step, we took the Fourier transform of the motion and look at the spectrum in Fig. 4.

The small peaks are due to the coupling of transverse motion while the large peak is the fractional part of the tune at a fixed intensity. Finally, the same analysis could be done for different beam intensities and the vertical tune of the centroid as a function of beam intensity is shown in Fig. 5.

The coherent tune shift is a result of the increase in intensity and it is given by $[1,2]$

$$
\Delta \nu_{z}=j \frac{e Z_{\perp}}{4 \pi R m \gamma \nu_{z} \omega^{2}} \hat{I}
$$

where $Z_{\perp}$ is the transverse impedance, $e$ is the proton charge, $\hat{I}=N_{\mathrm{B}} e /\left(\sqrt{2 \pi} \sigma_{t}\right)$ is the peak current, $\sigma_{t}$ is the 


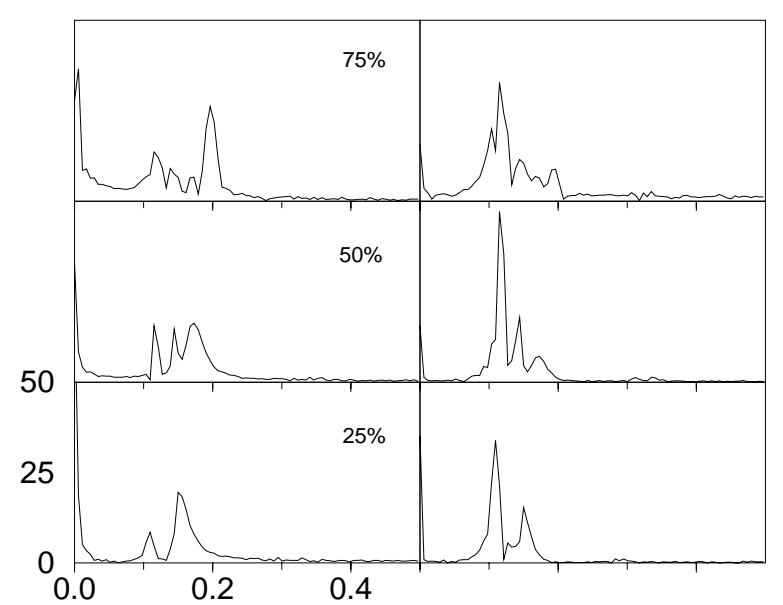

Figure 4: Amplitude of the Fourier transform of Fig. 3 versus fractional part of the tune. Left: horizontal. Right: vertical. Bottom to top: the head, the centroid and the tail of the beam. $N_{\mathrm{B}}=1.3 \times 10^{13}$.

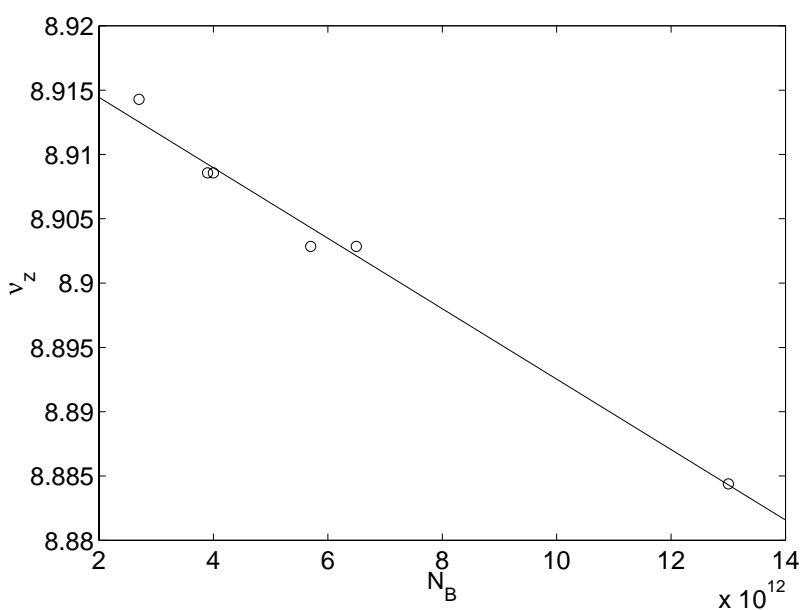

Figure 5: $\nu_{z}$ versus $N_{\mathrm{B}}$. Note the detuning due to the increase in the total number of proton $N_{\mathrm{B}}$ in a bunch. Circle: data for the vertical tune of the centroid(50\%) as a function of the beam intensity. Line: a fit by Eq. 2. Value of the slope $-2.739 \times 10^{-15}$ can be used to find the effective beam pipe radius $b$.

rms bunch length, $R$ is the mean radius of accelerator, $m$ the mass of proton, $\gamma=1 /\left(1-\beta^{2}\right)^{1 / 2}, j^{2}=-1, \nu_{z}$ is the vertical betatron tune, and $\omega$ is the revolution frequency. The most important contribution to $Z_{\perp}$ is due to the induced charge and current on the beam pipe and it is given by $[1,3]$

$$
Z_{\perp}=j \frac{Z_{0} R}{\gamma^{2} \beta^{2} b^{2}}
$$

where $Z_{0}=\mu_{0} c$ is the impedance of free space, $b$ is the beam pipe radius. Since the resistive wall impedance $(\sim$ $10^{5} \Omega / \mathrm{m}$ ) is an order of magnitude smaller than Eq. 3 and therefore it can be neglected. Using the slope of Fig. 5 and Eq. 2, $b$ can be estimated and it is found to be about $3.4 \mathrm{~cm}$. The actual shape of the beam pipe varies. However, most of it is nearly elliptical with vertical half aperture of $3.89 \mathrm{~cm}$ and horizontal half aperture of $8.66 \mathrm{~cm}$. Therefore, $b$ has the same order of magnitude of a few centimeters as the actual beam pipe dimensions. Since the cross section of the actual beam pipe is not circular, $b$ should be regarded as an effective size of the beam pipe.

\section{CONCLUSION}

The vertical betatron tune of the centroid decreased by 0.03 when the intensity increased from $2.7 \times 10^{12}$ to $13 \times 10^{12}$. The reasonable value of $b$ obtained by applying the circular beam pipe model indicates that the major source that caused the tune shift is the induced charge and current on the beam pipe. We also observed enhanced linear coupling at high space charge region (see the middle plots of Fig. 3).

\section{REFERENCES}

[1] S. Y. Lee, Accelerator Physics, (World Scientific, Singapore, 1999).

[2] J. Gareyte, Beam Observation and the Nature of Instabilities, AIP Conf. Proc. 184, 343-429 (Fermilab Summer School 1987).

[3] K. Y. Ng, Fields,Impedance and Structures, AIP Conf. Proc. 184, 472-524 (Fermilab Summer School 1987). 